\title{
Managing Aquatic Invasions: Optimal Locations and Operating Times for Watercraft Inspection Stations
}

\author{
Samuel M. Fischer ${ }^{1,}$, Martina Beck ${ }^{2}$, Leif-Matthias Herborg ${ }^{3}$, and Mark A. Lewis ${ }^{1,4}$ \\ ${ }^{1}$ Department of Mathematical and Statistical Sciences, University of Alberta, Edmonton, AB. \\ ${ }^{2}$ BC Ministry of Environment and Climate Change Strategy, Conservation Science Section, Victoria, BC. \\ ${ }^{3}$ Fisheries and Oceans Canada, Institute of Ocean Sciences, Sidney, BC. \\ ${ }^{4}$ Department of Biological Sciences, University of Alberta, Edmonton, AB. \\ * Department for Mathematical and Statistical Sciences; 632 Central Academic Building; University of Alberta; \\ Edmonton, AB; T6G 2G1; E-Mail: samuel.fischer@ualberta.ca
}

\begin{abstract}
Aquatic invasive species (AIS) cause significant ecological and economic damages around the world. A major spread mechanism for AIS is traffic of boaters transporting their watercraft from invaded to uninvaded waterbodies. To inhibit the spread of AIS, several Canadian provinces and American states set up watercraft inspection stations at roadsides, where potentially infested boats are screened for AIS and, if necessary, decontaminated. However, since budgets for AIS control are limited, watercraft inspection stations can only be operated at specific locations and daytimes. Though theoretical studies provide managers with general guidelines for AIS management, more specific results are needed to determine when and where watercraft inspections would be most effective. This is the subject of this paper. We show how linear integer programming techniques can be used to optimize watercraft inspection policies under budget constraints. We introduce our approach as a general framework and apply it to the prevention of the spread of zebra and quagga mussels (Dreissena spp.) to the Canadian province British Columbia. We consider a variety of scenarios and show how variations in budget constraints, propagule sources, and model uncertainty affect the optimal policy. Based on these results, we identify simple, generally applicable principles for optimal AIS management.
\end{abstract}

Keywords: aquatic invasive species; linear integer programming; optimal management; spatially explicit; zebra mussel 


\section{Introduction}

Human traffic and trade are major vectors for invasive species (Lockwood et al., 2013). Due to the significant ecological and economic damages invasive species cause (Pimentel et al., 2005), government regulations restrict the import of certain goods and require treatment of potentially infested freight and carriers (Shine et al., 2010; Johnson et al., 2017; Turbelin et al., 2017). While such regulations may be enforced comparatively easily at ports, air ports, and border crossings, control of inland traffic is more difficult, as a vast number of routes need to be monitored. This applies for example to the spread of zebra and quagga mussels (Dreissena spp.) and other aquatic invasive species (AIS), which often spread with watercraft and equipment transported from invaded to uninvaded waterbodies (Johnson et al., 2001). Zebra and quagga mussels are invasive in North America and have negative effects on native species and ecosystems, water quality, tourism, and infrastructure (Rosaen et al., 2012; Karatayev et al., 2015).

To counteract the spread of these AIS, watercraft inspection stations are set up on roads, where transported watercraft are inspected for AIS and decontaminated if at risk for carrying AIS (Mangin, 2011; Alberta Environment and Parks Fish and Wildlife Policy, 2015; Inter-Ministry Invasive Species Working Group, 2015). However, since budgets for inspections are limited, not all pathways can be monitored around the clock, and managers need to prioritize certain locations and daytimes. Though several theoretical studies provide managers with helpful guidelines for their work (Leung et al., 2002; Potapov and Lewis, 2008; Potapov et al., 2008; Vander Zanden and Olden, 2008; Finnoff et al., 2010; Hyytiäinen et al., 2013), more specific results are needed in practice to determine the locations and times where and when control is most effective. To date it has been difficult to tackle these questions rigorously, as comprehensive models for road traffic of potential vectors were missing. Therefore, AIS managers have relied on past watercraft inspection data, shared experience between jurisdictions, and iterative improvements of control policies. Recent modelling advances (Fischer et al., 2019), however, now permit the application of quantitative methods to optimize control measures in road networks and to evaluate their effectiveness. This will be the subject of this paper.

Our goal will be to minimize the number of boaters reaching uninvaded waterbodies without 
being inspected for AIS. Thereby, we will assume that a fixed budget is available for AIS control. This problem setup differs from scenarios considered in other studies on optimal control of invasive species (Hastings et al., 2006; Potapov and Lewis, 2008; Potapov et al., 2008; Finnoff et al., 2010; Epanchin-Niell and Wilen, 2012), where budget allocation over time is optimized along with the control actions. However, to optimize the budget, invasions need to be assigned "cost labels". This is an often difficult and politically sensitive task. Furthermore, the budget available for AIS control may be subject to political and social influences and determined on a different decision hierarchy than the management actions. Therefore, AIS managers may seek to spend a fixed yearly budget optimally rather than to determine the theoretically best control budget. The presence of fixed budget constraints also reduces the need to consider the invasion as a dynamic process.

Identifying the locations where a maximal number of boaters could be screened for AIS is similar to the problem of finding optimal locations for road-side infrastructure (Trullols et al., 2010). A well-known technique to solve such problems is linear integer programming (Conforti et al., 2014). The idea is to model the optimization problem with functions linear in the decision variables. Though solving linear integer programs is a computationally difficult task in general, good approximate solutions can often be determined, and a variety of software tools are available to compute solutions. Therefore, linear integer programming has also been used in the context of invasive species management (Epanchin-Niell and Wilen, 2012; Kıbış and Büyüktahtakın, 2017).

A crucial step in linear integer optimization is to find a problem formulation that facilitates good approximations (Ageev and Sviridenko, 2004). In this paper, we provide such a formulation to optimize locations and operating times of watercraft inspection stations. This problem differs from comparable resource allocation problems (Surkov et al., 2008; Trullols et al., 2010), as we need to account for the temporal variations in traffic. These variations are key when we consider the trade-off between operating few inspection stations intensely, e.g. around the clock, and distributing resources over many locations operated at peak traffic times only.

We demonstrate the potential of our approach by applying it to optimize watercraft inspection policies for the Canadian province British Columbia (BC). We show how uncertainty, different cost constraints, and additional propagule sources impact the optimal policy. Thereby, we identify 
control principles applicable beyond the considered scenario.

This paper is structured as follows: we start by introducing model components required to optimize watercraft inspection station operation. Then, we show how the considered optimization task can be formulated as linear integer problem. Thereby, we focus first solely on inspection station placement before we introduce the full problem, in which also operating times of inspection stations are optimized. After this general description of our approach, we apply the method to AIS management in BC and present results under different scenarios. Lastly, we discuss our results and the limitations of our approach and draw general conclusions on AIS management.

\section{Method}

\section{$2.1 \quad$ Model}

Our goal is to identify how limited resources can be allocated most effectively to minimize the number of boaters arriving at uninvaded waterbodies without being inspected for AIS. We assume that two aspects of the control strategy can be changed: the locations and operating times of watercraft inspection stations. As traffic typically follows cyclic patterns, we consider one such cycle as the time horizon for the control optimization.

To find an optimal inspection policy, we need three models (see Figure 1): (1) a model for boater traffic, (2) a model for control, and (3) a model for control costs. The traffic model gives us estimates of when, where, and along which routes boaters travel. The control model shows us when and where inspections could be conducted and how effective they are. Lastly, the cost model measures the costs for inspections. The information from the three models serve as input for a control optimizer that determines a good - or, if possible, the best - watercraft inspection strategy. Below, we describe each of the models in greater detail before we introduce suitable optimization routines in the next section. 


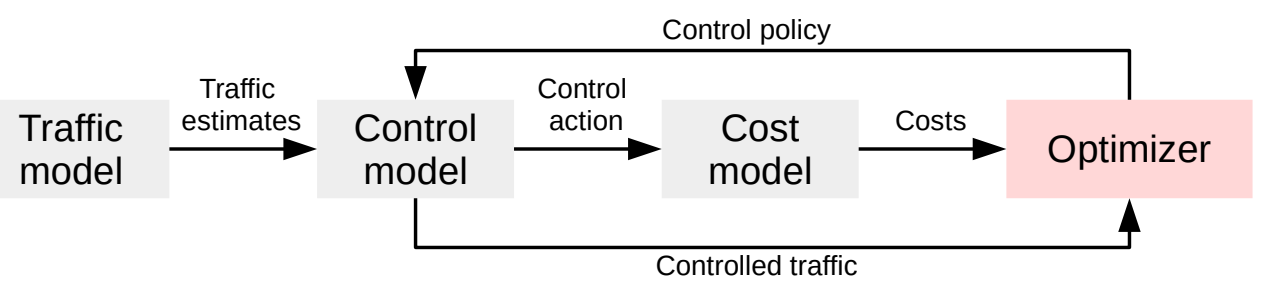

Figure 1: Components of our approach. The control model determines how the traffic estimated by the traffic model changes under a given control policy. The cost model yields the costs for control actions. The optimizer maximizes the controlled traffic subject to a cost constraint.

\subsubsection{Traffic model}

The traffic model provides estimates of when, where, and along which routes boaters drive. Knowledge about routes is key to understanding whether boaters passing one control location have already been inspected at another location. For each considered route, the traffic model provides us with a traffic estimate. In this study, we use a hybrid gravity and route choice model (Fischer et al., 2019) to estimate the traffic. The model includes components accounting for boaters' travel incentive, their route choice, the timing of traffic, and boaters' compliance with inspections.

In practice it is rarely feasible to consider all routes that boaters could possibly take, and we need to focus on some set of "reasonable" routes (Bovy, 2009; Fischer, 2019). As a consequence, there may be some agents travelling along unexpected routes. When boaters travelling along such routes arrive at inspection locations, we do not know whether their watercraft have been inspected earlier. This makes it difficult to optimize inspection strategies. Nonetheless, we may want to account for these boaters by introducing a "noise" term to our model. To that end, we assume that a fraction of the travelling boaters could be observed at any inspection location with a small probability (see Fischer et al., 2019).

As road traffic is rarely uniform over time, we furthermore need a submodel predicting how traffic varies with time. While it may be comparatively easy to determine the temporal distribution of traffic at a specific location, it can be difficult to identify the temporal relationship between traffic at two locations on the same route. For example, agents passing one location in the morning may not be able to reach another location before the afternoon. Modelling such relationships is particularly difficult for locations far from each other, as boaters may have different travel speeds. 
We therefore apply a simplification and assume all boaters travelling along a route have the same speed.

\subsubsection{Control model}

We assume that there is a specific set of locations where watercraft inspections could be conducted. For example, these locations could be pullouts large enough to provide a safe environment for inspections. We suppose that compliant boaters stop for an inspection whenever they pass an operated inspection station. Conversely, uncompliant boaters are assumed to bypass any inspection station on their route. Consequently, we seek to maximize the number of boaters that pass at least one operated watercraft inspection.

As with the inspection locations, we assume that there are specific time intervals when inspection can be conducted. The admissible time intervals may be determined by safety concerns or practical considerations and can be location dependent. As staff cannot move between distant inspection locations easily, and the working hours of inspection staff are subject to legal and practical constraints, we may furthermore assume that every inspection station can be operated in shifts of given lengths only.

\subsubsection{Cost model}

Inspection costs may be split in two classes: infrastructure costs that apply once for each chosen inspection location, and operational costs, which depend on when and for how long an inspection station is operated. The operational costs may also account for ongoing equipment maintenance costs and training of staff. The control costs may be location and time dependent. For example, it may be expensive to conduct inspections at remote locations if staff must travel long distances to their work place. Furthermore, some locations will require significantly more infrastructure costs (e.g. lighting and washrooms) in order to operate overnight shifts. In addition, wages are often higher in overnight shifts. 


\subsection{Optimizing control locations}

With the submodels from the previous section at hand, we can proceed optimizing the inspection strategy. Optimizing both locations and operating times of watercraft inspection stations at the same time is conceptually and computationally challenging. To ease the introduction of our approach, we first consider a scenario in which inspection stations are operational around the clock. In this case, we can ignore the temporal variations of traffic and focus on choosing optimal control locations (cf. Trullols et al., 2010).

In this section, we show how the corresponding optimization problem can be formulated as a linear integer problem. To that end, we let $L$ be the set of all admissible inspection locations and introduce for each location $l \in L$ a binary variable $x_{l}$ that assumes the value 1 if and only if an inspection station is set up at $l$. Let $R$ be the set of potential routes that boaters may choose, $n_{r}$

the expected number of complying boaters travelling along route $r \in R$, and $L_{r} \subseteq L$ the set of locations where the boaters travelling on route $r$ could be inspected.

As noted earlier, one inspection station suffices to control all complying boaters driving along a route $r$. Consequently, boaters travelling on route $r$ will be controlled if and only if

$$
\sum_{l \in L_{r}} x_{l} \geq 1
$$

Otherwise, the left hand side of equation (1) will be 0 . Therefore, we can express the total number of inspected boaters by

$$
F_{\text {loc }}(\boldsymbol{x}):=\sum_{r \in R} \min \left\{1, \sum_{l \in L_{r}} x_{l}\right\} n_{r} .
$$

To formulate the cost constraint, let $c_{l}$ be the cost for operating control location $l \in L$ and $B$ the available budget. As we assume that all inspection stations are operated for the same time, we do not need to distinguish between infrastructure and operation costs. Hence, we can write the 
cost constraint as

$$
\sum_{l \in L} c_{l} x_{l} \leq B
$$

The optimal placement policy can be identified by maximizing $F_{\text {loc }}(\boldsymbol{x})$ over all $\boldsymbol{x} \in\{0,1\}^{|L|}$ subject to constraint (3). Though $F_{\text {loc }}$ contains a "minimum" function, $F_{\text {loc }}$ can be easily transformed to a linear function by introducing further variables and linear inequality constraints (see e.g. Ageev and Sviridenko, 1999). Since the left hand side of the cost constraint (3) is linear in $\boldsymbol{x}$ as well, and $\boldsymbol{x}$ is constrained to be a vector of integers, the considered optimization problem is a linear integer problem. This can be solved with a suitable general linear integer programming solver or a specifically tailored rounding algorithm (Ageev and Sviridenko, 2004). We discuss possible optimization routines in section 2.5.

\subsection{Optimizing control locations and timing}

After focusing on inspection station placement, we now extend our approach to permit free choice of inspection station operating times. In this extended scenario, we need to balance the trade-off between operating few highly frequented inspection stations around the clock and distributing efforts over many locations operated at peak traffic times only. This trade-off makes combined optimization of location choice and timing more challenging than separate optimization of location choice and timing (cf. Epanchin-Niell and Wilen, 2012).

While location choice is a discrete optimization problem - each potential inspection location is either chosen or not - optimization of operating times is a continuous problem, since inspections could be started at any time. To exploit the toolset of discrete optimization anyway, we simplify our problem by discretizing time. That is, we split the boater traffic corresponding to boaters' departure times and consider only discrete sets of admissible inspection shifts.

Let $T$ be a set of disjunct time intervals covering the complete time span of interest. We write $n_{r t}$ for the expected number of boaters who travel on route $r \in R$, start their journey in time interval $t \in T$, and are willing to comply with inspections. Let furthermore $S_{l}$ be the set 
of admissible inspection shifts for location $l \in L$. Each shift corresponds to a time interval in which the inspection station is operated. Since the shift lengths are given, the set $S_{l}$ can be fully characterized by the shifts' start times.

As we assume that all boaters travelling along a route have the same speed, we can determine the set $S_{l r t} \subseteq S_{l}$ of control shifts during which boaters who started their journey in time interval $t \in T$ arrive at location $l \in L$ via route $r \in R$. Under reasonable error allowance, it is usually possible to construct the sets $S_{l r t}$ in a way that each shift covers the departure time intervals either completely or not at all, respectively. This setup prevents issues arising if some intervals overlap only partially.

To formulate our optimization problem as linear integer problem, we describe the control policy again with binary variables $x_{l s} \in\{0,1\}$. Here, $x_{l s}$ is 1 if and only if an inspection station at location $l \in L$ is operated in shift $s \in S_{\text {lrs }}$. Agents travelling on route $r \in R$ who departed in time interval $t \in T$ are controlled if and only if

$$
\sum_{l \in L_{r}} \sum_{s \in S_{l r t}} x_{l s} \geq 1
$$

Consequently, the total controlled agent flow is given by

$$
F_{\text {full }}(\boldsymbol{x}):=\sum_{r \in R} \sum_{t \in T} \min \left\{1, \sum_{l \in L_{r}} \sum_{s \in S_{l r t}} x_{l s}\right\} n_{r t} .
$$

To derive the cost constraint, recall that we distinguish between infrastructure costs $c_{l}^{\text {loc }}$ for using location $l$ and operating $\operatorname{costs} c_{l s}^{\text {shift }}$ payable per control shift $s$ conducted at $l$. Consequently, the total costs for control at $l$ are given by

$$
\sum_{s \in S_{l}} c_{l s}^{\text {shift }} x_{l s}+c_{l}^{\text {loc }} \max _{r \in R, t \in T}\left(\sum_{s \in S_{l r t}} x_{l s}\right)
$$


and the cost constraint reads

$$
\sum_{l \in L}\left(\sum_{s \in S_{l}} c_{l s}^{\text {shift }} x_{l s}+c_{l}^{\text {loc }} \max _{r \in R, t \in T}\left(\sum_{s \in S_{l r t}} x_{l s}\right)\right) \leq B
$$

As in the previous section, $B$ denotes the available budget. Optimizing $F_{\text {full }}$ subject to $(7)$ is a linear integer problem, since the "minimum" term in (5) and the "maximum" terms in (7) can be replaced by introducing correspondingly constrained variables.

\subsection{Noise}

Even if the traffic model accounts for most routes boaters use, some boaters may travel along unexpected routes. It is difficult to optimize inspection station operation with regards to these boaters, as we do not know which inspection stations cover the same routes. Nonetheless, it can be desirable to account for noise, since the level of uncertainty may affect the optimal inspection policy.

In the absence of a mechanistic model for traffic noise, we may assume that boaters who are travelling on unexpected routes are passing any inspection location with a small probability $\eta_{o}$, whereby they choose the passing time randomly. Under this assumption, the expected number of inspected boaters travelling along unknown routes is given by

$$
F_{\text {noise }}=\left(1-\prod_{l \in L}\left(1-\eta_{o} \sum_{s \in S_{l}} x_{l s} \tau_{s l}\right)\right) n_{\text {noise }} .
$$

Here, $n_{\text {noise }}$ denotes the expected number of boaters travelling on unknown routes.

As $F_{\text {noise }}$ is not a convex function, adding this noise term to the objective function would make optimization difficult. However, as $\eta_{0}$ is typically small, equation (8) is well approximated by

$$
\hat{F}_{\text {noise }}=\eta_{o} n_{\text {noise }} \sum_{l \in L} \sum_{s \in S_{l}} x_{l s} \tau_{s l}
$$

which is linear and can thus be easily added to the linear integer problem. This approximation is most precise if $x_{l s}=0$ for most $l$ and $s$. If the budget is high enough to operate many inspection 
stations for long times, the noise may be overestimated. However, since $n_{\text {noise }}$ is typically small compared to the total boater traffic, inaccuracies in the noise model are unlikely to alter the overall optimization results significantly.

\subsection{Solving the optimization problems}

Having derived the problem formulation in the previous sections, we now proceed by discussing suitable solution methods. The inspection station placement problem described in section 2.2 is equivalent to the budgeted maximum coverage problem (Khuller et al., 1999), also called maximum coverage problem with knapsack constraint (Ageev and Sviridenko, 2004). This problem is well studied in computing science, and it has been shown that finding a solution better than factor $\left(1-e^{-1}\right)$ of the optimum is an NP-hard, often infeasibly difficult, problem (Feige, 1998). This result applies also to the extended problem introduced in section 2.3, as it is more general than the placement problem. Though these theoretical results show that scenarios exist in which the problems considered in this paper cannot be solved exactly within reasonable time, good approximate or even optimal solutions can often be obtained in practical applications.

When seeking a good solution, we can exploit that the linear integer formulation of our problem helps us to obtain upper and lower bounds to solutions efficiently. Consider a slightly changed optimization problem in which the management variables $\boldsymbol{x}$ are not constrained to be integers but drawn from the continuous domain $[0,1]^{N}$. Here, $N$ is the dimension of the problem. In this case, the problems can be solved with linear programming techniques within seconds even if $N$ is large. Clearly, the integer domain $\{0,1\}^{N}$ is a subset of the continuous domain $[0,1]^{N}$. Therefore, the solution to the problem with relaxed integer constraint is an upper bound to the desired integer solution.

Often it is possible to obtain good integer solutions by rounding the solution to the continuous problem. Ageev and Sviridenko (2004) present an algorithm that always achieves the approxima-

tion bound $\left(1-e^{-1}\right)$ for the inspection station placement problem, in which operating times are fixed. Nonetheless, general solvers with possibly poorer worst-case performance may yield better solutions in "benign" cases. A number of generally applicable methods exist (Conforti et al., 2014). 
In this study, we use branch and bound methods, in which the distance between upper and lower bounds on the optimal objective are found by solving continuously relaxed subproblems with some constrained variables.

A challenge that general solvers face is to find a good initial feasible solution that they can

improve on. For the pure inspection station placement problem, we could apply the rounding algorithm by Ageev and Sviridenko (2004), which would also guarantee us the best approximation bound. However, for the joint optimization of both placement and operating times of watercraft inspection station, we are not aware of any algorithm with such a guarantee. We therefore propose a "greedy" rounding algorithm to obtain good initial solutions. The idea is to solve the relaxed linear programming problem and to determine the largest non-integer decision variable that can be rounded up without violating the cost constraint. We applied this procedure with some improvements described in Supplementary Appendix A. In applications, we consistently obtained solutions better than $80 \%$ of the optimum with this approach.

\section{Application}

To show the potential of our approach, we applied it to optimize watercraft inspections in the Canadian province British Columbia (BC). Below we provide an overview of the scenario-specific submodels we used. Furthermore, we briefly describe our implementation of the presented approach.

\subsection{Scenario-specific submodels}

\subsubsection{Traffic model}

To model boater traffic, we used the hierarchical gravity and route choice model for boater traffic presented in Fischer et al. (2019). The model was fitted to data collected at British Columbian watercraft inspection stations in the years 2015 and 2016. At the time this study was conducted, dreissenid mussels were not known to be established anywhere in BC. As sources of potentially infested boaters, we therefore considered the Canadian provinces and American states that (1) 
were known to be invaded by dreissenid mussels or (2) had connected waterway to an infested jurisdiction and no coordinated mussel detection program in place at the time the data were collected. As sinks we identified 5981 potentially boater accessible lakes in BC.

To estimate the boater traffic between an origin and destination, the model considered characteristics of the donor jurisdiction, the recipient lake, and the distance between the two. Major sources of high-risk boaters were characterized by high population counts. Furthermore, Canadian provinces were found to have higher boater traffic to BC than American states. Attractiveness of destination lakes increased with their surface area, the population counts of surrounding towns and cities, and the availability of close-by touristic facilities, such as campgrounds. Lastly, the boater flow was estimated to decay in cubic order of the distance between an origin and a destination. For a detailed description of the model along with precise parameter estimates, refer to Fischer et al. (2019).

To identify potential boater pathways, we computed locally optimal routes (Fischer, 2019) between the considered origins and destinations. These routes arise if routing decisions on local scales are rational and based on simple criteria (here: minimizing travel time) whereas unknown factors may affect routing decisions on larger scales. Consequently, the model accounts for routes arising from a multitude of mechanisms. The attractiveness of the routes was computed based on their length measured in travel time. Again, a more in-depth description of the model and the fitted parameter values can be found in Fischer et al. (2019).

The fraction of boaters travelling on routes not covered by our traffic model was estimated as 4.9\%. However, this number is not estimable from survey data obtained at watercraft inspection stations, because it is negatively correlated with the parameter $\eta_{o}$ (section 2.4 ), denoting the probability to observe a boater travelling on an unknown route at an arbitrary inspection location. Therefore, Fischer et al. (2019) introduced an additional model assumption bounding the noise term below $5 \%$. Note that due to the dependency of $\eta_{o}$ on the noise level, the estimability issue has little effect on the noise level observed at watercraft inspection stations and thus on inspection policy. Based on a noise level of 4.9\%, $\eta_{o}$ was estimated as 0.06 (Fischer et al., 2019).

The temporal distribution of traffic was modelled with a von Mises distribution. This is a 
unimodal circular distribution often used in models (Lee, 2010). The temporal pattern was assumed to have a period of one day. The traffic high was estimated to be at $2 \mathrm{PM}$, whereby the estimated peak traffic was 15 times higher than the estimated traffic volume at night. As traffic data were available for specific inspection locations only, we assume that the temporal traffic distribution is uniform over all locations.

Assuming an equal temporal traffic distribution for all potential inspection locations makes it difficult to account for the time boaters need to travel between two sites. This, is a model limitation but not of major concern in the considered scenario of boater traffic to BC. First, note that we seek locations that are not on the same pathway. If boaters do not pass multiple operated inspection locations, we are safe to neglect the travel time between sites. Furthermore, we can exploit that the considered boater origins are located outside of the province and boaters drive, with minor exceptions, along highways in one particular direction. Consequently, the temporal traffic distribution of close-by locations on such a highway would be equal up to a shifting term, and the optimized inspection times could be adjusted accordingly.

\subsubsection{Control model}

As described in section 2.1.2, we assume that every complying boater passing an operated inspection location is inspected for invasive mussels. The compliance rate across all inspection stations was estimated to be $80 \%$ (Fischer et al., 2019). To find potentially suitable locations for inspections, we identified pullouts across BC. We reduced the number of possible options by disregarding some pullouts in close proximity to others. In total, we considered 249 location candidates.

Due to the large number of location candidates, we did not conduct a detailed evaluation of the operational suitability of all considered locations (e.g. pullout size, signage, and safety). Instead, we consulted with the BC Invasive Mussel Defence Program to gauge the general suitability of the locations suggested by the optimizer. If a suggested location seemed unsuitable, we removed it from the candidate set and repeated the optimization procedure. Despite this superficial suitability check, a more detailed analysis would be necessary to account for all potential practical constraints. These must be considered independent of the model before an inspection station can be placed. 
For each location, we assumed that $8 \mathrm{~h}$ long inspection shifts could be started at each full hour of the day. Note that "shift" here refers to the time inspections are conducted and does not include time required for staff to access or set up an inspection station. The work time of staff will therefore be longer in practice. The assumed length of the inspection shifts aligns with average operation patterns of watercraft inspection stations in $\mathrm{BC}$ and divides each day in three equally sized shifts, which simplifies the model. Though the effective operation time (limited by access time of staff) is lower at remote locations, our time model provides a good first approximation.

\subsubsection{Cost model}

We determined the inspection costs based on correspondence with the BC Invasive Mussel Defence Program. The considered optimization problem is often easier to solve if costs are rounded to well aligned cost units. Therefore, we set the infrastructure costs for setting up an inspection station as our base cost unit. The costs per conducted inspection shift are then 3.5 units during daytime hours and 5.5 units between $9 \mathrm{PM}$ and $5 \mathrm{AM}$. These costs include salary, training, and equipment for inspection staff. In 2017, the BC Invasive Mussel Defence Program was operating on a budget of approximately 80 cost units.

As in-depth location-specific cost analysis would have been difficult, we assumed that the inspection costs are equal for all considered locations. Note, however, that site specific costs can vary significantly and may be a limitation when assessing a location for overnight operations.

\subsection{Implementation}

As we considered about 300, 000 origin-destination pairs connected by 6.7 routes on average, considering all boater pathways individually would be difficult. Therefore, we merged traffic of boaters passing the same sets of potential inspection locations. As a result, the number of distinct boater flows reduced to 2026 .

We determined the optimal inspection locations and operating times under different budget scenarios. This allowed us to determine the budget required to minimize the fraction of uninspected high-risk boaters to a desired level. We also varied the model's noise term to test how inspection 
strategies change under increased uncertainty. To see how new infestations in close-by jurisdictions change the inspection policy, we furthermore considered a scenario in which the American states Idaho, Wyoming, and Oregon are invaded.

We implemented the model in the high-level programming language Python version 3.7. To formulate the linear integer problem, we used the modelling software CVXPY version 1.0.25 with added support for initial guesses. To solve the linear integer problem, we used the commercial solver MOSEK. We computed initial guesses with the greedy rounding procedure described in section 2.5. We let the solver terminate if a solution with guaranteed accuracy of $99.5 \%$ was found or if 50 minutes had passed. We conducted the computations on a Linux server with a 20 core Intel Xeon 640 E5-2689 CPU (3.1GHz per CPU) and with 512GB RAM. The computer code can be retrieved from vemomoto.github.io.

\section{Results}

In $72 \%$ of the considered scenarios, we were able to identify a solution with the desired accuracy of 99.5\%. In the remaining cases, the guaranteed solution quality never fell below 92\%; in scenarios with budgets $B \geq 25$ units, we could always identify solutions with $98 \%$ accuracy and above. The greedy algorithm used to compute an initial guess provided a solution with $99.5 \%$ accuracy in $58 \%$ of the considered cases. The initial guesses always had a quality above $90 \%$.

Figure 2 displays the optimized locations and operating times for watercraft inspection stations in the considered model scenario. We depict the respective optimal policy under three different budget constraints. The optimal locations for inspections are located close to border crossings if suitable locations are available. However, where the traffic through many border crossings merges on a major highway (e.g. in the Vancouver metropolitan area), it is optimal to place the inspection stations farther inland.

Figure 3 depicts characteristics of the optimal inspection stations in different scenarios. The expected traffic volume at an inspection station coincides with the optimized operating times: stations with high expected boater traffic are operated longer than stations with lower traffic. If the budget is increased, some stations are assigned longer operating times. However, larger 


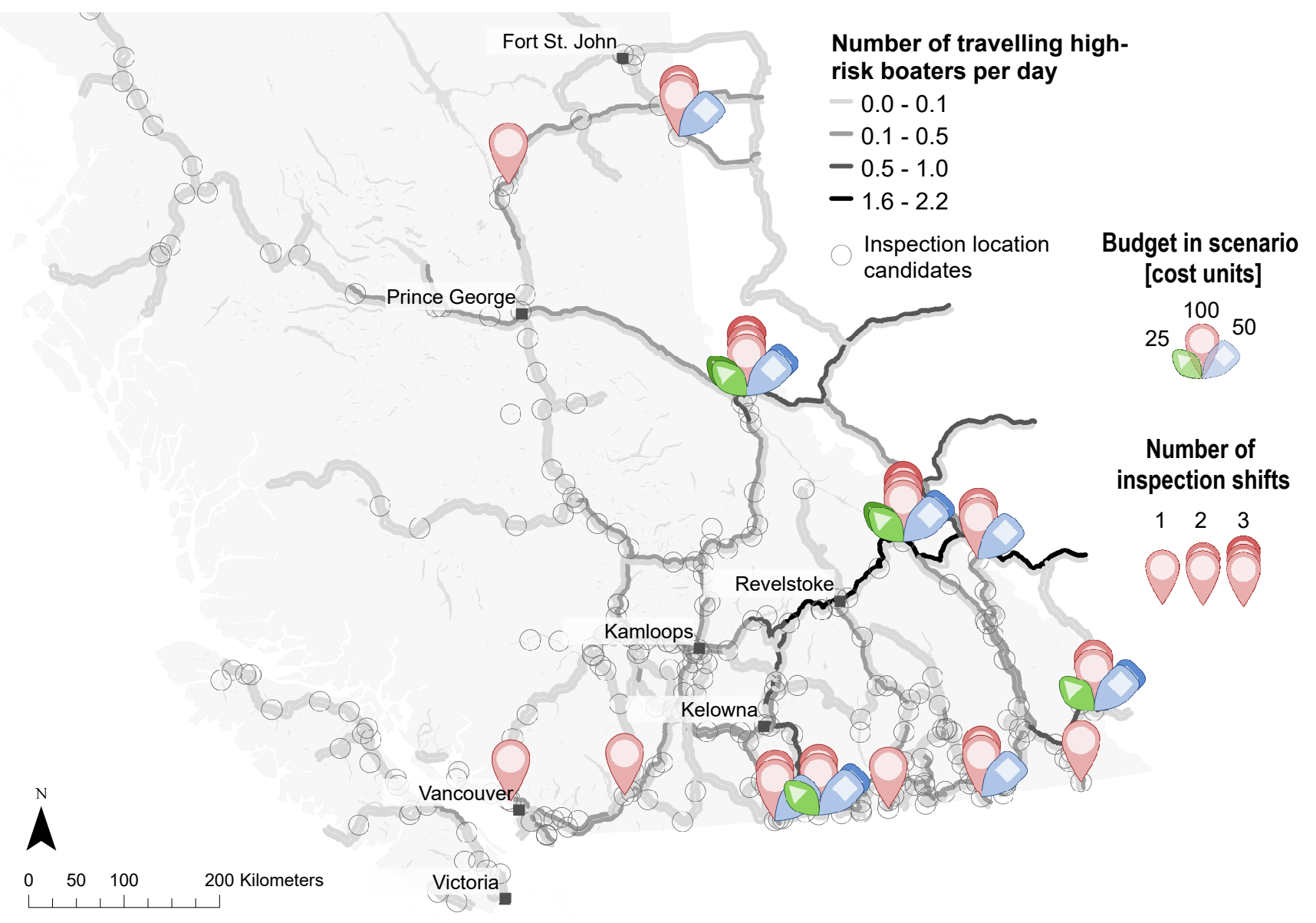

Figure 2: Optimal locations and operation shifts for three different budget scenarios. Most inspection stations are placed close to the British Columbian border. The markers depict the optimal inspection locations for each scenario. Green (triangle): optimal locations with a budget of 25 units; blue (square) 50 unit budget; red (circle) 100 unit budget. The number of markers stacked on top of each other corresponds to the optimal numbers of inspection shifts. The darkness of the roads show the estimated boater traffic volume. The hollow circles depict the considered candidates for inspection locations. 
(a)
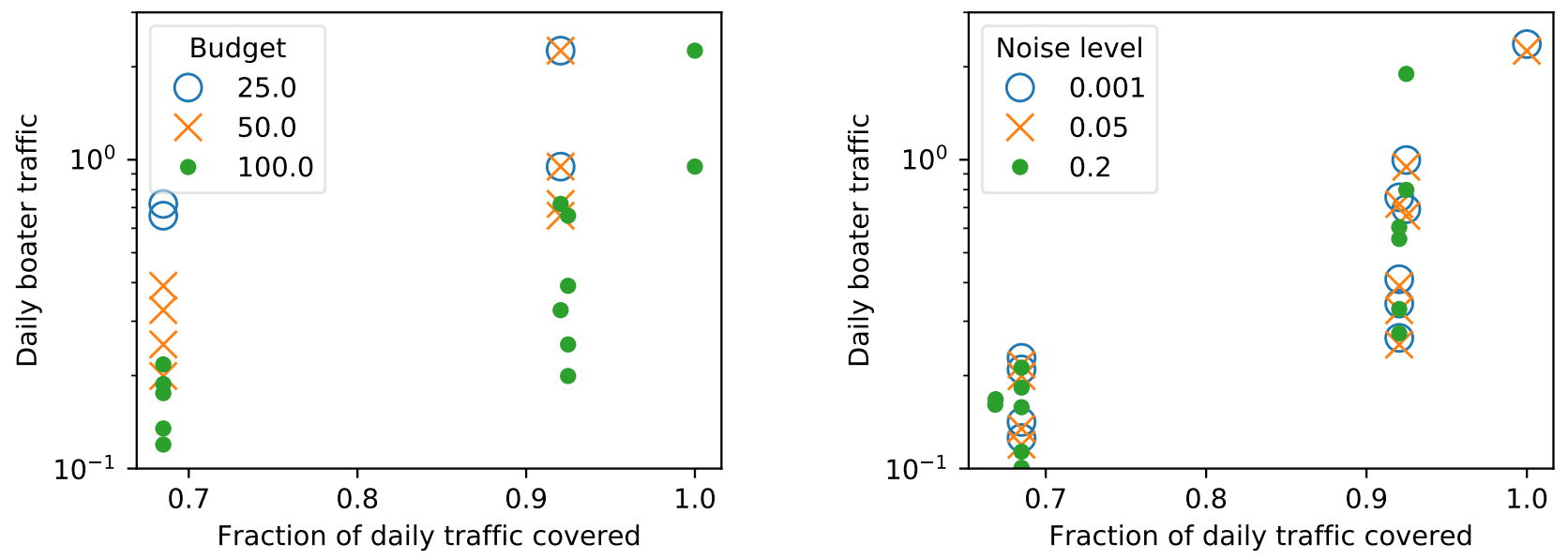

Figure 3: Characteristics of the optimized inspection stations in scenarios with (a) different budget constraints, and (b) different levels of uncertainty. Additional budget is preferably spent on additional inspection locations rather than longer operating hours. Increased uncertainty results in resources being distributed over more locations at cost of decreasing operating hours. Overall, however, uncertainty does not have a strong effect on the inspection policy. Each marker corresponds to an inspection station. The position of a marker depicts the daily traffic volume expected at the location and the fraction of daily traffic covered under the optimal operation policy (compliance supposed). The "noise level" denotes the fraction $\eta_{c}$ of boaters travelling on routes not covered by the route choice model. Note that the noise level also affects the daily traffic volume at the inspection locations.

portions of the additional budget are spent on additional locations (see also Figure 2). If the uncertainty in the traffic predictions increases, more inspection stations are set up at the cost of shorter operations. Overall, however, the noise level has little effect on the inspection policy.

Optimizing inspection station operation under a range of different budget allowances showed that a moderate inspection budget, corresponding to about half the 2017 BC inspection budget, suffices to inspect half of the incoming high-risk boaters (Figure 4). However, the resources required for inspections increase quickly if more boaters shall be controlled. Thereby, the faction of inspected boaters is limited by boaters' compliance with inspections.

The considered change in the invasion state of three American states had only a moderate impact on inspection policy. The results are depicted in Supplementary Appendix B. As the additional propagule sources were located south of $\mathrm{BC}$, the inspection effort increased at the southern border under the optimal policy. Furthermore, the optimal policy contained less overnight inspections and distributed resources more evenly across inspection stations. 
(a)
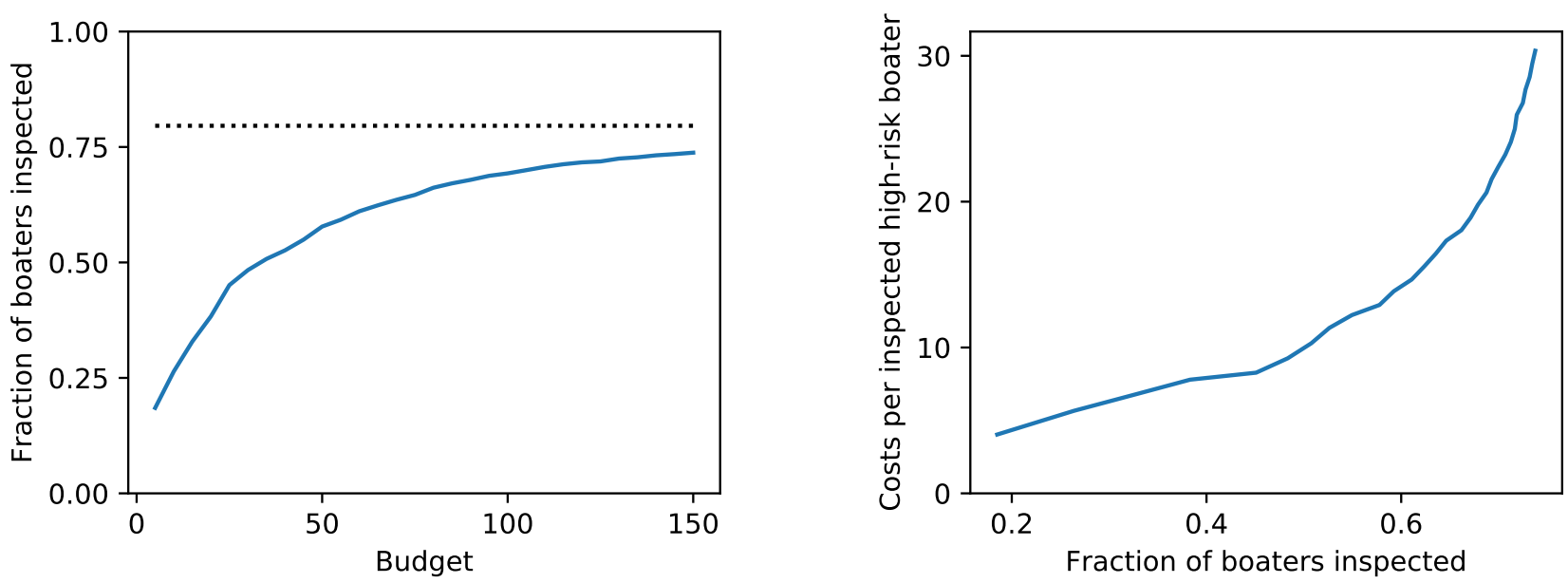

Figure 4: Inspection effectiveness dependent on the budget constraint (a) and price per inspected high-risk boater dependent on the proportion of inspected boaters (b). While a large fraction of high-risk boaters can be covered with moderate effort, inspecting all complying boaters is costly. Panel (a) shows the expected fraction of incoming high-risk boaters that can be inspected under the optimal policy. The dotted line shows the level of complying boaters, which is the maximal fraction of boaters that can be inspected.

\section{Discussion}

We presented a method to optimize placement and operating times of watercraft inspection stations. The approach is suited to model management scenarios on a detailed level and gives specific advice for management actions. We applied our approach to invasive mussel management in BC and investigated the impact of budget constraints, model uncertainty, and potential future invasions on management actions and efficiency. However, it must be recognized that our model did not account for all critical operational factors, such as site safety. Nonetheless, the presented results provide valuable insights into optimal management of AIS when combined with critical operational factors.

Most of our results are consistent with common sense. In general, it is optimal to inspect boaters as soon as they enter the managed region. That way, waterbodies close to the border can be protected. If multiple routes via different border crossings merge close to the border, it can be optimal to inspect boaters after this merging point. Inspections stations should operate longer at locations with high traffic volume. Furthermore, uncertainty in traffic predictions increases the 
benefit of spreading the inspection efforts over many locations. Driven by these simple principles, our results were remarkably robust throughout considered scenarios and agree well with the watercraft inspection policy currently implemented in BC.

While these qualitative principles may seem obvious, it can be challenging to identify quantitative definitions of terms like "close to the border" and "longer". The difficulty in optimizing management policies is in balancing trade-offs, such as between leaving some waterbodies close to the border unprotected and maximizing the overall number of inspected boaters, or between long-time operation of few highly frequented inspection stations and distribution of resources over many locations. As the approach proposed in this paper is suited to account for these trade-offs, it is a valuable extension to earlier more theoretical results on AIS management (Potapov and Lewis, 2008; Potapov et al., 2008; Finnoff et al., 2010).

Considering scenarios with different budget constraints allowed us to investigate the tradeoff between resources invested in AIS control and the number of inspected high-risk boaters. In combination with the expected monetary damage caused by the arrival of an uncontrolled boater at an uninvaded lake, this trade-off curve can be used to identify the optimal budget for inspections. Since both invasion risk and damages due to invasions are difficult to quantify, a rigorous computation of the optimal inspection budget may not always be feasible in practice. Nonetheless, the cost-effectiveness curve provides an estimate of the efficacy of control efforts and shows which budget is required to achieve a certain management goal.

In the case of AIS control in $\mathrm{BC}$, a moderate budget suffices to inspect a significant portion of the incoming high-risk boaters. This is because boater traffic in $\mathrm{BC}$ concentrates on a small number of major highways. Nevertheless, an attempt to inspect all high-risk boaters would be very costly, as many minor roads would have to be considered as well. It could therefore be more cost-effective to implement measures to increase the compliance of boaters, e.g. through additional road signs or public outreach and education.

We see particular use of our approach in its potential to optimize rapid response actions under scenarios of interest. The extended invasion scenario considered in this paper shows that slight adjustments to the inspection policy may suffice to react on the new conditions. In a similar 
manner, our approach could be used to assess the benefit from cross-border collaborations, in which inspection efforts are combined to control the boater inflow to a large joint area. Due to the flexibility of our model, managers can consider a variety of scenarios at little cost.

\subsection{Limitations and possible extensions}

The accuracy of our approach in real-world applications is strongly dependent on the accuracy and level of detail of the utilized data and models. Therefore, the results should be combined with expert knowledge and refined iteratively if necessary. Nonetheless, our approach can be extended to account for many management constraints and is thus a helpful tool to optimize inspection policies.

Limitations exist with respect to the considered objective function. Though the number of potentially infested watercraft arriving at a waterbody is a valuable proxy for invasion risk, the establishment probability of dreissenid mussels is not linear in propagule pressure (Leung et al., 2004). Hence, our approach is not suited to minimize invasion risk directly. However, highdimensional non-convex optimization problems are difficult to solve, and minimizing a proxy for invasion risk may thus be the better option in practice. Nonetheless, significant realism could be added by considering the suitability of the destination waterbodies as habitat for AIS. This could be done by weighting boater flows differently dependent on the invasion risk of the destination waterbodies.

Since our traffic model does not explicitly account for the time boaters need to travel between locations, the optimized inspection station operating times may have to be adjusted to local temporal traffic patterns. Though this shortage in model realism could affect the results significantly if boaters pass multiple inspection stations under the optimal policy, optimal inspection locations are often on independent routes. In the scenario considered in this study, the optimized operating times were all centered around the traffic peak. This indicates that interactions between locations did not affect the operating times and the error due to the simplifying model assumption is small.

Another modelling challenge is to account for uncertainty appropriately. The noise model used in this study is a non-informative null model that treats all potential inspection locations equally. 
As more boater traffic may be expected at major highways than at minor roads, the noise model could be improved by incorporating location-dependent covariates. However, since our results were not very sensitive to the noise level, a realistic noise model might not change the optimal policy significantly.

Our model did not incorporate site-specific costs and operational constraints. In high-budget scenarios, this let our model suggest overnight inspections at remote sites that are lacking the required infrastructure to safely operate at night. Requirements for overnight inspections include proper road infrastructure (lanes/barriers), lighting, access to safe communication and nearby living accommodations for staff. A lack of living accommodations for staff can also limit the number of staff based in remote locations. These constraints could be incorporated in a more detailed model as well as increased costs at remote locations. A more detailed model could also account for inspection stations operated by neighbouring jurisdictions. As an example, the BC program works closely with the Canadian Border Services Agency and neighbouring provinces and states to receive advanced notifications of high risk watercraft destined for BC. Nonetheless, the presented model includes major factors affecting inspection station operation. Therefore, the model can serve as a helpful resource to inform managers' decisions in parallel with operational constraints.

Another potential extension of our model is to incorporate location-specific or managementdependent compliance rates. At certain sites, such as cross-national border crossings, compliance can be enforced more easily than at other locations. Compliance may furthermore depend on management efforts: it may be possible to increase the compliance rate of boaters at some costs. In Supplementary Appendix C, we show how non-uniform and flexible compliance rates can be considered with small model adjustments.

The computational method we used to optimize inspection station operation is well established and builds on a large body of theoretical insights (Ageev and Sviridenko, 2004; Conforti et al., 2014). Nonetheless, the problem is computational difficult, and there may be scenarios in which linear integer solvers fail to provide good solutions. Optimization failures are most prevalent in scenarios in which portions of the budget remain unused under the optimal policy or in which many 
boaters pass multiple inspection stations under optimized operation. In both cases, the solution to the continuous relaxation of the problem may differ significantly from the integer solution.

However, issues due to unused budget become minor if the considered budget is sufficiently large. Furthermore, the issue may be mitigated by adjusting the budget slightly. Issues with redundant inspection stations, in turn, are unlikely to occur if the propagule donors and recipients are in separate regions. Then, independent inspection locations can often be identified. This is often the case if invasion processes are considered on large scales. Therefore, our approach will yield good results in most applications. We provide more details in Supplementary Appendix D.

\subsection{General conclusions for invasive species management}

In this paper, we considered specific management scenarios with focus of AIS control in BC. Nonetheless, some common patterns were consistent throughout our results and may thus apply with greater generality. These principles may be used as rules of thumb if no comprehensive modelling and optimization effort is possible. Below we summarize these conclusions.

- Inspection stations should be placed close to the border of the uninfested region. Consequently, cross-border collaborations between uninvaded jurisdictions have a high potential of improving the cost-effectiveness of control.

- If traffic flows merge close to the border, inspections are more cost-effective after the merging point. Hence, identifying such points is crucial for successful management.

- If traffic predictions involve a high level of uncertainty, inspection efforts should be distributed over many locations at the cost lower inspection effort at each site.

- If a high reduction of the propagule inflow is desired, it may be most cost-effective to implement measures increasing the compliance rate rather than operating more inspection stations for longer hours. 


\section{Authors' contributions}

All authors conceived the project; SMF conceived the methods jointly with MAL. SMF and MB jointly prepared the data for the analysis. SMF conducted the mathematical analysis, implemented the model, and wrote the manuscript. All authors revised the manuscript.

\section{Acknowledgements}

The authors would like to give thanks to the BC Ministry of Environment and Climate Change Strategy staff of the BC Invasive Mussel Defence Program, who conducted the survey this study is based on. Furthermore, the authors thank the members of the Lewis Research Group at the University of Alberta for helpful feedback and discussions. SMF is thankful for the funding received from the Canadian Aquatic Invasive Species Network and the Natural Sciences and Engineering Research Council of Canada (NSERC); MAL gratefully acknowledges an NSERC Discovery Grant and Canada Research Chair.

\section{Competing Interests}

The authors declare no competing interests.

\section{References}

Ageev, A. and Sviridenko, M. (2004). Pipage rounding: a new method of constructing algorithms with proven performance guarantee. Journal of Combinatorial Optimization, 8(3):307-328.

Ageev, A. A. and Sviridenko, M. I. (1999). Approximation algorithms for maximum coverage and max cut with given sizes of parts. In Cornuéjols, G., Burkard, R. E., and Woeginger, 
G. J., editors, Integer Programming and Combinatorial Optimization, volume 1610, pages 1730. Springer Berlin Heidelberg, Berlin, Heidelberg.

Alberta Environment and Parks Fish and Wildlife Policy (2015). Alberta Aquatic Invasive Species Program 2015 annual report. Technical report, Edmonton, AB.

Bovy, P. H. L. (2009). On modelling route choice sets in transportation networks: a synthesis. Transport Reviews, 29(1):43-68.

Conforti, M., Cornuéjols, G., and Zambelli, G. (2014). Integer programming, volume 271 of Graduate Texts in Mathematics. Springer International Publishing, Cham.

Epanchin-Niell, R. S. and Wilen, J. E. (2012). Optimal spatial control of biological invasions. Journal of Environmental Economics and Management, 63(2):260-270.

Feige, U. (1998). A threshold of $\ln \mathrm{n}$ for approximating set cover. Journal of the ACM, 45(4):634652.

Finnoff, D., Potapov, A., and Lewis, M. A. (2010). Control and the management of a spreading invader. Resource and Energy Economics, 32(4):534-550.

Fischer, S. M. (2019). Locally optimal routes for route choice sets. arXiv e-prints, pages 1-40. arXiv:1909.08801.

Fischer, S. M., Beck, M., Herborg, L.-M., and Lewis, M. A. (2019). A hybrid gravity and route choice model to assess vector traffic in large-scale road networks. arXiv e-prints, pages 1-26. arXiv:1909.08811.

Hastings, A., Hall, R. J., and Taylor, C. M. (2006). A simple approach to optimal control of invasive species. Theoretical Population Biology, 70(4):431-435.

Hyytiäinen, K., Lehtiniemi, M., Niemi, J. K., and Tikka, K. (2013). An optimization framework for addressing aquatic invasive species. Ecological Economics, 91:69-79.

Inter-Ministry Invasive Species Working Group (2015). Zebra and quagga mussel early detection and rapid response plan. Technical report, Victoria, BC. 
Johnson, L. E., Ricciardi, A., and Carlton, J. T. (2001). Overland dispersal of aquatic invasive species: a risk assessment of transient recreational boating. Ecological Applications, 11(6):17891799.

Johnson, R., Crafton, R. E., and Upton, H. F. (2017). Invasive species: major laws and the role of selected federal agencies. Technical report, Congressional Research Service, Washington, DC.

Karatayev, A. Y., Burlakova, L. E., and Padilla, D. K. (2015). Zebra versus quagga mussels: a review of their spread, population dynamics, and ecosystem impacts. Hydrobiologia, 746(1):97112 .

Khuller, S., Moss, A., and Naor, J. S. (1999). The budgeted maximum coverage problem. Information Processing Letters, 70(1):39-45.

Kıbış, E. Y. and Büyüktahtakın, İ. E. (2017). Optimizing invasive species management: a mixedinteger linear programming approach. European Journal of Operational Research, 259(1):308321.

Lee, A. (2010). Circular data. Wiley Interdisciplinary Reviews: Computational Statistics, 2(4):477486.

Leung, B., Drake, J. M., and Lodge, D. M. (2004). Predicting invasions: Propagule pressure and the gravity of Allee effects. Ecology, 85(6):1651-1660.

Leung, B., Lodge, D. M., Finnoff, D., Shogren, J. F., Lewis, M. A., and Lamberti, G. (2002). An ounce of prevention or a pound of cure: bioeconomic risk analysis of invasive species. Proceedings of the Royal Society B: Biological Sciences, 269(1508):2407-2413.

Lockwood, J. L., Hoopes, M. F., and Marchetti, M. P. (2013). Invasion ecology. Wiley-Blackwell, Chichester, West Sussex, UK, 2nd edition.

Mangin, S. (2011). The 100th Meridian Initiative: a strategic approach to prevent the westward spread of zebra mussels and other aquatic nuisance species. Technical Report 152, U.S. Fish and Wildlife Service, Arlington, VA. 
Pimentel, D., Zuniga, R., and Morrison, D. (2005). Update on the environmental and economic costs associated with alien-invasive species in the United States. Ecological Economics, $52(3): 273-288$.

Potapov, A. B. and Lewis, M. A. (2008). Allee effect and control of lake system invasion. Bulletin of Mathematical Biology, 70(5):1371-1397.

Potapov, A. B., Lewis, M. A., and Finnoff, D. C. (2008). Optimal control of biological invasions in lake networks. Natural Resource Modeling, 20(3):351-379.

Rosaen, A. L., Grover, E. A., and Spencer, C. W. (2012). The costs of aquatic invasive species to Great Lakes states. Technical report, Anderson Economical Group, East Lansing, MI.

Shine, C., Kettunen, M., Genovesi, P., Essl, F., Gollasch, S., Rabitsch, W., Scalera, R., Starfinger, U., and ten Brink, P. (2010). Assessment to support continued development of the EU Strategy to combat invasive alien species. Final Report for the European Commission, Institute for European Environmental Policy (IEEP), Brussels, Belgium.

Surkov, I. V., Oude Lansink, A. G. J. M., van Kooten, O., and van der Werf, W. (2008). A model of optimal import phytosanitary inspection under capacity constraint. Agricultural Economics, $38(3): 363-373$.

Trullols, O., Fiore, M., Casetti, C., Chiasserini, C., and Barcelo Ordinas, J. (2010). Planning roadside infrastructure for information dissemination in intelligent transportation systems. Computer Communications, 33(4):432-442.

Turbelin, A. J., Malamud, B. D., and Francis, R. A. (2017). Mapping the global state of invasive alien species: patterns of invasion and policy responses. Global Ecology and Biogeography, 26(1):78-92.

Vander Zanden, M. J. and Olden, J. D. (2008). A management framework for preventing the secondary spread of aquatic invasive species. Canadian Journal of Fisheries and Aquatic Sciences, $65(7): 1512-1522$. 


\title{
Supplementary Appendices for "Managing Aquatic \\ Invasions: Optimal Locations and Operating Times for
} Watercraft Inspection Stations"

\author{
Samuel M. Fischer ${ }^{1, *}$, Martina Beck², Leif-Matthias Herborg ${ }^{3}$, and Mark A. \\ Lewis $^{1,4}$ \\ ${ }^{1}$ Department of Mathematical and Statistical Sciences, University of Alberta, Edmonton, AB. \\ ${ }^{2}$ BC Ministry of Environment and Climate Change Strategy, Conservation Science Section, Victoria, \\ $B C$. \\ ${ }^{3}$ Fisheries and Oceans Canada, Institute of Ocean Sciences, Sidney, BC. \\ ${ }^{4}$ Department of Biological Sciences, University of Alberta, Edmonton, AB. \\ *samuel.fischer@ualberta.ca
}

\section{A Greedy rounding algorithm}

In this Appendix, we describe the greedy rounding algorithm we applied to obtain initial guesses for the general branch and bound solvers. We start by introducing some helpful notation. Let $P$ be the linear integer problem that we desire to solve and $P_{\text {cont }}$ its continuous relaxation, in which decision variables may attain fractional values. We write $\boldsymbol{x}$ for the $N$-dimensional vector of decision variables, indexed by $(l, s) \in L \times S$. Let $\boldsymbol{e}_{l s}$ be a unit vector that is 0 everywhere except for the component corresponding to the index $(l, s)$. Suppose that $C(\boldsymbol{x})$ denotes the cost for implementing a policy given by $\boldsymbol{x}$. We provide pseudo code for the greedy rounding algorithm in Algorithm 1. 
The algorithm repeatedly solves the relaxed problem $P_{\text {cont }}$ with different constraints fixing some decision variables to integer values. The algorithm proceeds in two phases. In the first phase, the maximal non-integral decision variable that can be rounded up without violating the budget constraint is determined. With this variable fixed, problem $P_{\text {cont }}$ is solved again. When no additional component can be rounded up without violating the cost constraint, all previous constraints are removed, and the set of utilized locations is fixed instead. The algorithm sets a flag locked to True to show that the second phase of the algorithm has started.

In the second phase, components of $\boldsymbol{x}$ are still rounded up if possible. However, now we do not round up the largest non-integral component of $\boldsymbol{x}$. Instead, we determine for some location $l \in L$ with non-integral operation (i.e. $\exists \tilde{s} \in S_{l}: x_{l \tilde{s}} \notin\{0,1\}$ ) the first time interval

$$
t:=\underset{t \in T}{\operatorname{minargmax}}\left\{\sum_{s \in S_{l t}} x_{l s} \mid x_{l s}<1 \forall s \in S_{l t}\right\}
$$

that is operated strongest at this location. Here, minargmax $\{\cdot\}$ refers to the minimal admissible value for $\operatorname{argmax}\{\cdot\}$ if the maximum is not unique. Then, we round up the latest affordable shift $s \in S_{l}$ that covers the time interval $t$ and add $x_{l s}=1$ to the set of constraints. If no additional shift can be operated at location $l$, we add a constraint fixing the usage of this location: $x_{l s}=\left\lfloor x_{l s}\right\rfloor$ for all $s \in S_{l}$.

Distinguishing between the two phases of the algorithm yields optimized operating times. Suppose we are in phase 2, and consider the example depicted in Figure A2. The solution to the relaxed problem $P_{\text {cont }}$ suggests that 3 inspection shifts $s_{1}, s_{2}$, and $s_{3}$ are conducted fractionally at the considered location $l$. Thereby, $s_{2}$ overlaps with $s_{1}$ and $s_{3}$. The respective operation intensities are $x_{l_{1}}=x_{s_{3}}=0.8$ and $x_{l_{2}}=0.2$. The budget assigned to this location does not suffice to operate both $s_{1}$ and $s_{3}$ completely. Hence, only one shift can be operated at $l$. Naive greedy rounding would suggest to operate shift $s_{1}$, as it is the earliest shift with the maximal fractional operation. However, in the optimal solution, the time interval between 8 AM and 4 PM should be operated strongest. Therefore, shift $s_{2}$ would be the optimal choice.

In its second phase, the suggested algorithm rounds up shifts based on the maximal cumulative operation rather than choosing the shift with the highest operation variable. Nonetheless, it would 


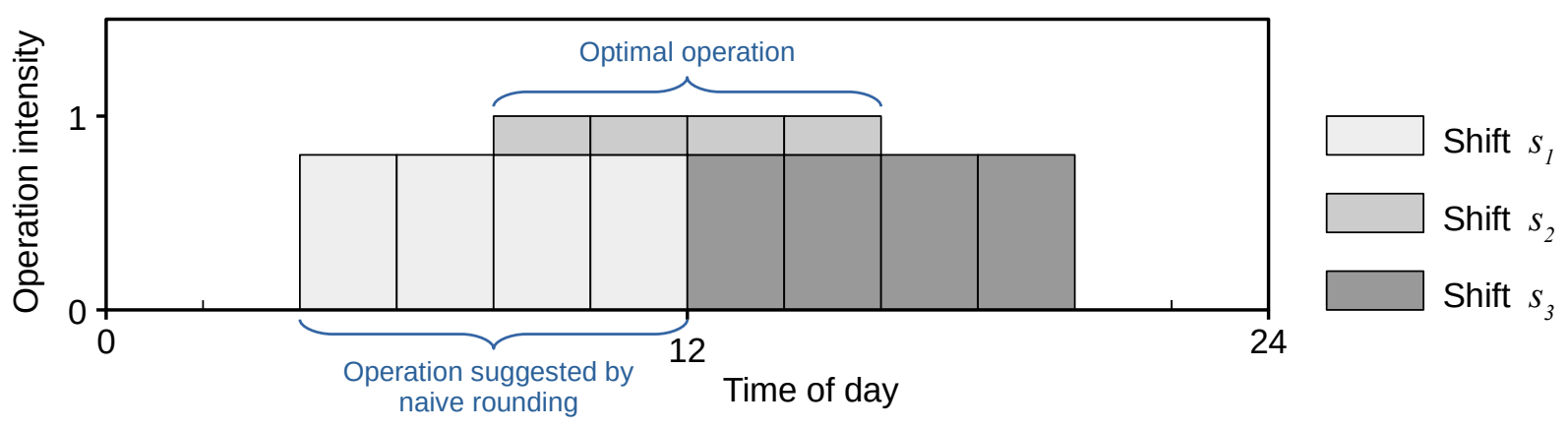

Figure A1: Motivation for the changed rounding procedure in phase 2 of the greedy rounding algorithm. The operation intensity is depicted as a function of time for some inspection location. The intervals on the time axis depict the discretization of the day time. The grey boxes show the extent to which the inspection station would be operated in the respective time intervals if fractional operation would be allowed. The boxes' colours correspond to the respective operation shifts. Naive greedy rounding would suggest to operate shift $s_{1}$. Improved rounding, however, would prefer the time interval in which the cumulative operation is maximal ( shift $\left.s_{2}\right)$.

be of disadvantage to apply this rounding scheme in phase 1 of the algorithm, in which the set of used locations is not fixed. In this case, shifts in the middle of the day would always be chosen with preference, which make operation of two shifts on a day less efficient. In the second phase, it is typically known how many shifts should be operated at each location.

Slight improvements to the suggested algorithm are possible. For example, we added constraints in phase 1 to suppress fractional operation of shifts that would not be affordable completely under the costs of the already constrained variables. However, this improvement is unlikely to have a major effect on the results.

\section{B Optimal inspection policy if additional parts of the USA are infested}

To assess how the optimal inspection policy changes if additional states are infested, we considered a scenario in which boaters from Idaho, Oregon, and Wyoming were considered high-risk boaters. The results are depicted in Figure A2. As more high-risk boaters enter BC via the southern border, inspection efforts at this border are increased. The required resources are freed by operating fewer inspection stations over night and by abandoning inspection locations in the north. Nonetheless, 


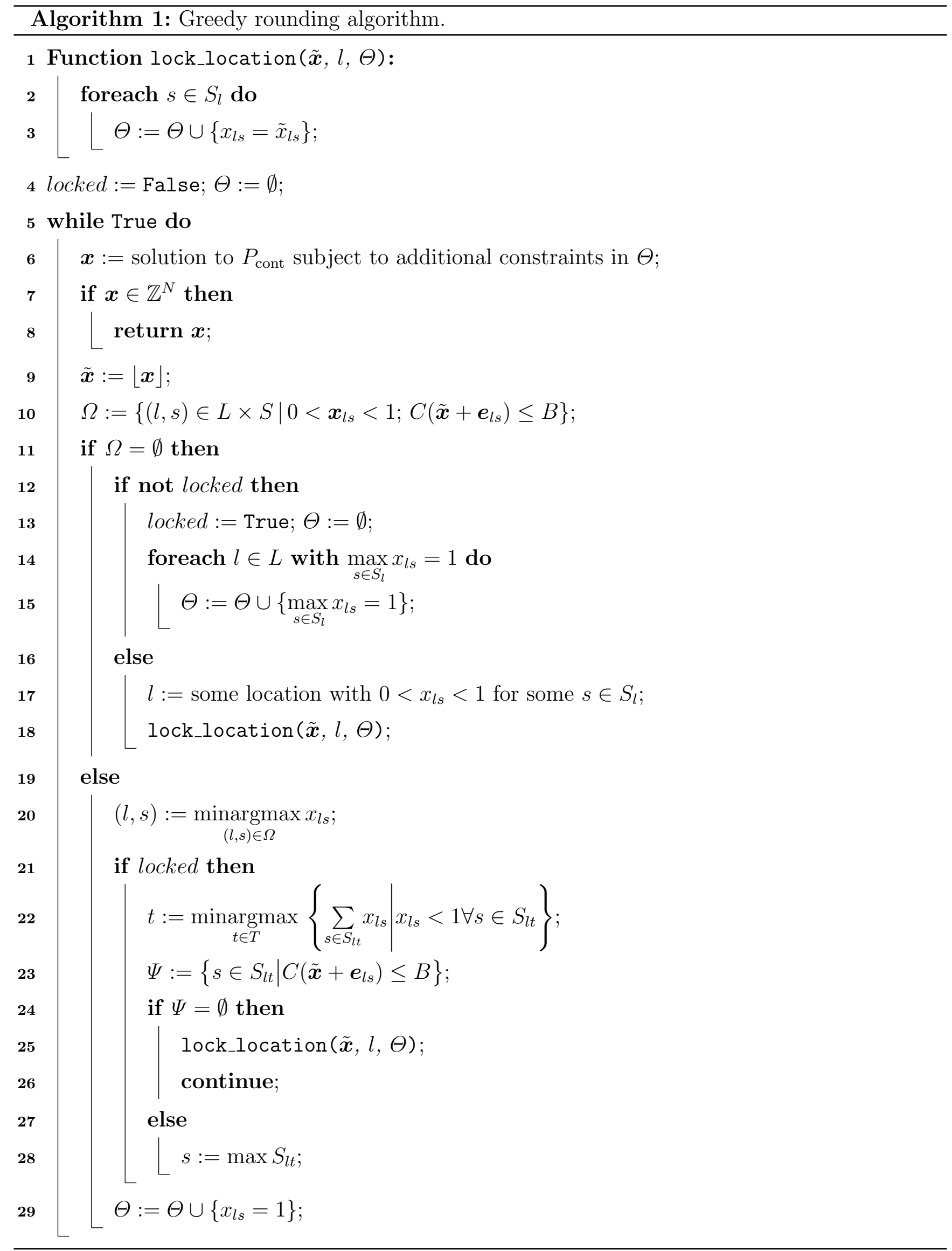




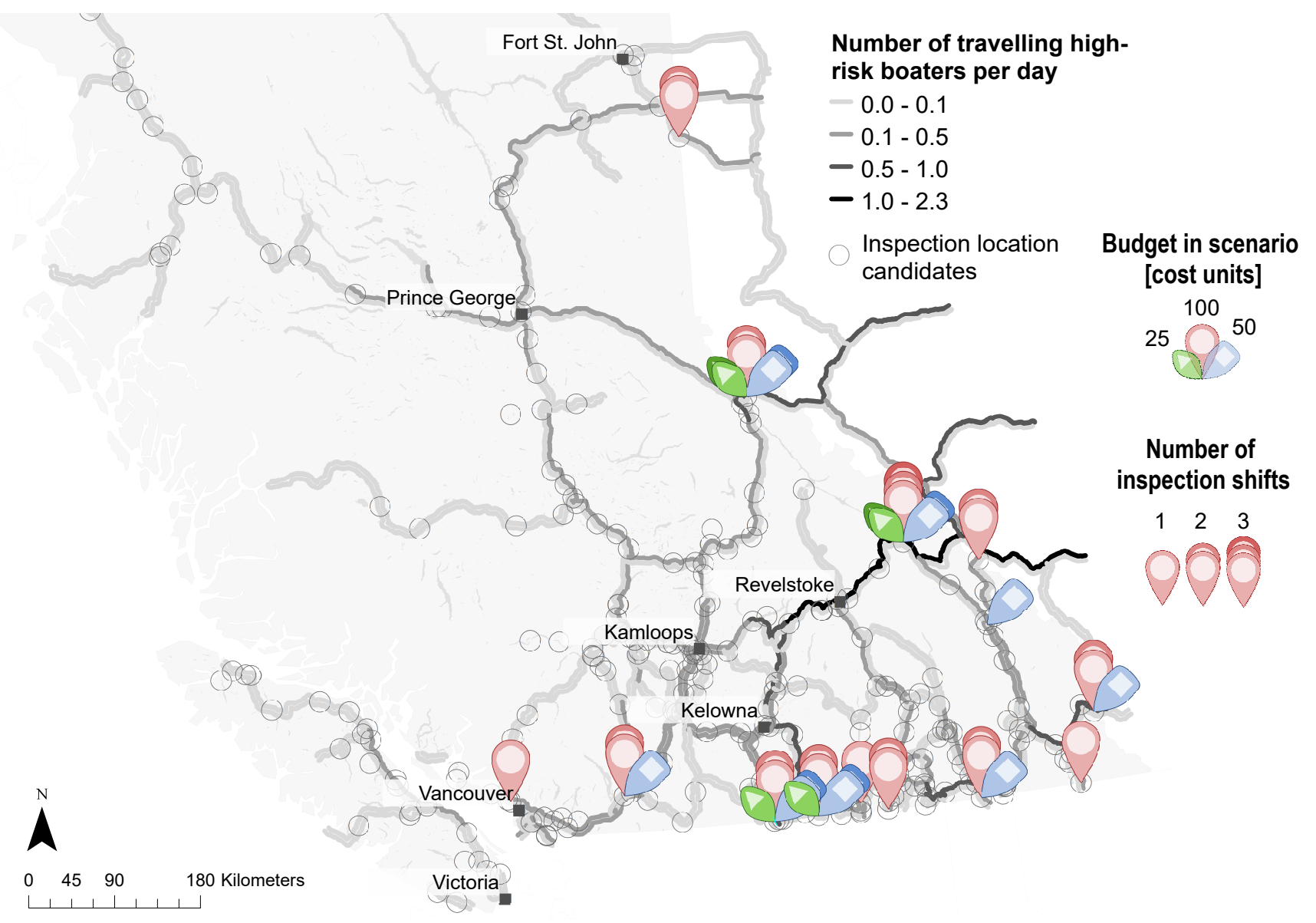

Figure A2: Optimal locations and operation shifts assuming that Idaho, Oregon, and Wyoming are mussel invaded. Compared to the base scenario with fewer infested States south of BC, more inspections are conducted at the southern border of the province. The symbols have the same meaning as in Figure 2 (main text).

the overall changes are moderate, because even in the changed invasion scenario most high-risk boaters are expected to enter the province via the eastern border.

\section{Flexible and location-specific compliance rates}

It may be more cost-effective to implement measures enforcing boaters' compliance than to operate many inspection stations for long hours. Furthermore, compliance of boaters may be higher or enforced more easily at some specific locations. In this appendix, we show how the approach presented in this paper can be adjusted to take these factors into account. 


\section{C.1 Location-specific compliance rates}

We start by considering the case of non-uniform compliance rates. To that end, we split the boater flows based on the compliance of the boaters. Let $C$ be the set of possible compliance rates, $c_{l} \in C$ the expected compliance rate of boaters at location $l \in L$, and $L_{c}$ the set of locations with compliance rate $\tilde{c} \geq c$. For a route $r \in R$ and a time interval $t \in T$ Let $n_{r t c}$ be the expected number of boaters who travel along route $r \in R$, started their journey in time interval $t \in T$, and comply at all inspection locations $l$ with $l_{c} \geq c$ but not at inspection locations with $l_{c}<c$. These boaters will be inspected if and only if

$$
\sum_{l \in L_{r} \cap L_{c}} \sum_{s \in S_{l r t}} x_{l s} \geq 1
$$

As in the main text, $x_{l s}$ is a binary variable denoting whether inspections are conducted at location $l \in L$ in shift $s \in S$. Consequently, the total number of inspected boaters is given by

$$
F_{\text {loc-compliance }}(\boldsymbol{x}):=\sum_{c \in C} \sum_{r \in R} \sum_{t \in T} \min \left\{1, \sum_{l \in L_{r} \cap L_{c} s \in S_{l r t}} x_{l s}\right\} n_{r t c} \text {. }
$$

This function can be optimized with the same method discussed in the main text. With a similar approach, time-dependent compliance rates could be incorporated, too.

\section{C.2 Flexible compliance rates}

In some applications, the compliance rate may be altered at a specific cost. If these costs can be expressed as a convex function of the achieved compliance rate, a flexible compliance rate can be incorporated in our model easily. Below, we consider for simplicity the base case with a uniform compliance rate at all locations. Allowing location-specific flexible compliance rates can be done by combining the two approaches introduced in this appendix.

Let $n_{r t}$ be the expected number of boaters travelling on route $r \in R$ and who started their journey in time interval $t \in T$. Note that other than in the main text, compliance of these boaters 
is not supposed. Altering equation (5) from the main text to

$$
F_{\text {flex-compliance }}(\boldsymbol{x}):=c \sum_{r \in R} \sum_{t \in T} \min \left\{1, \sum_{l \in L_{r}} \sum_{s \in S_{l r t}} x_{l s}\right\} n_{r t}
$$

accounts for the flexible compliance rate $c$.

Let us assume assume that the costs for enforcing a specific compliance rate $c$ at a location $l \in L$ and during shift $s \in S$ are given by the linear function

$$
\operatorname{cost}_{l s}(c)=\alpha_{l}\left(c-c_{0}\right)
$$

whereby $c_{0}$ is the base compliance rate if no actions are taken to increase compliance. More complex cost functions can be modelled with convex piece-wise linear functions or general convex functions. Adding these costs to the overall cost function changes the cost constraint to

$$
\sum_{l \in L}\left(\sum_{s \in S_{l}}\left(c_{l s}^{\text {shift }}+\alpha_{l}\left(c-c_{0}\right)\right) x_{l s}+c_{l}^{\text {loc }} \max _{r \in R, t \in T}\left(\sum_{s \in S_{l r t}} x_{l s}\right)\right) \leq B
$$

In addition to changing the objective function and the cost constraint, we have to introduce one further constraint limiting the compliance rate to the feasible range:

$$
c_{0} \leq c \leq 1
$$

With these changes, the compliance rate can be optimized along with the inspection locations and operating times.

\section{Difficult inspection optimization scenarios}

In many real-world instances, good solutions to the linear integer problems derived in this paper can be identified within reasonable time. Nonetheless there are examples in which the optimization is computationally challenging. In this appendix, we discuss two important mechanism that can 
make it difficult to find a highly optimal solution in short time. We also provide examples for the discussed mechanisms.

Difficulties can arise (1) if a significant fraction of the budget is unused under the optimal policy and (2) if many boaters pass multiple operated inspection locations under the optimal policy. We start by considering budget-related issues before we discuss problems arising from unfavourable relationships between potential inspection locations. At the end of this appendix we discuss why these challenges are not of major concern in many real-world applications. To simplify explanations, we consider the case of optimizing inspection station placement only. The described mechanisms extend easily to the full problem in which operating times must be optimized as well.

\section{D.1 Difficulties due to cost constraints}

Let us first consider a scenario in which a fraction of the given budget remains unused under the optimal policy. For example, suppose that operation of an inspection station costs 5 cost units and that we are given a budget of 9 units. Consequently, 4 cost units of the budget will remain unused. To obtain an approximate solution and obtain an upper bound on the optimal objective value, solvers consider the problem's continuous relaxation, in which partial use of inspection locations (and shifts) is permissible. In this relaxed scenario, all 9 cost units will be spent, which allows the inspection of more boaters than in the realistic scenario with binary choices. Consequently, the upper bound on the solution given by the solution to the relaxed problem may be much higher than the true optimal solution. This makes it difficult to check whether an identified solution is highly optimal and thus increases computation time.

The problem described above becomes even more difficult if control actions with different costs are possible. Suppose that we may operate one of three inspection stations, which are passed by different sets of boaters, respectively. That is, no boater passes two of the potential inspection locations. Assume that per day $n_{1}=n_{2}=5$ boaters pass stations $l_{1}$ and $l_{2}$, respectively, and that $n_{3}=8$ boaters may be inspected at location 3 . Suppose we are given a budget of 9 units and that the costs for operating stations $l_{1}$ and $l_{2}$ are $c_{1}=c_{2}=5$ cost units, whereas operation of station $l_{3}$ requires $c_{3}=9$ cost units. 
Again, optimizers may consider the problem's continuous relaxation to find an approximate solution and a quality estimate. An optimal solution to the relaxed problem is to operate both station 1 and station 2 fractionally with weight $x_{1}=x_{2}=0.9$. Then, the total costs $x_{1} c_{1}+x_{2} c_{2}=9$ satisfy the budget constraint and the total number of inspected boaters is given by $x_{1} n_{1}+x_{2} n_{2}=9$. However, in the original integer problem, stations cannot be operated fractionally, and only one station can be chosen. As more boaters pass location 3 than locations $l_{1}$ or $l_{2}$, it would be optimal to conduct inspections at location $l_{3}$, where 8 boaters can be inspected. Applying a greedy rounding algorithm to the relaxed solution, however, would suggest to operate either location $l_{1}$ or $l_{2}$, where only 5 boaters would be expected.

\section{D.2 Difficulties due to unfavourable relations between inspection lo- cations}

Besides challenges induced by cost constraints, specific relationships between potential inspection locations can make the optimization difficult. Consider the example depicted in Figure A3, whereby an arbitrary number of boaters may drive from each origin/destination (black circle) to each other origin/destination. Suppose that operating an inspection location at any of the permissible locations has unit cost and that we are provided a budget of 2 cost units. If the relaxed version of the problem is considered and fractional operation of stations is permitted, operating each location

with intensity $\frac{1}{2}$ would cover all boater flows and hence be the optimal solution. However, if discrete choices must be made, some boaters will not be inspected. As all locations are operated equally in the optimal solution to the relaxed problem, this solution does not provide any hint towards which of the locations should be operated in the original scenario with binary decisions. Therefore, the problem is difficult to solve.

\section{D.3 Prevalence of difficult scenarios in real-world applications}

Any of the challenging scenarios discussed above can occur in real-world problems. However, certain characteristics of real-world scenarios lower the risk of running into optimization issues. In many management scenarios of interest, various inspection stations can be operated. Problems 


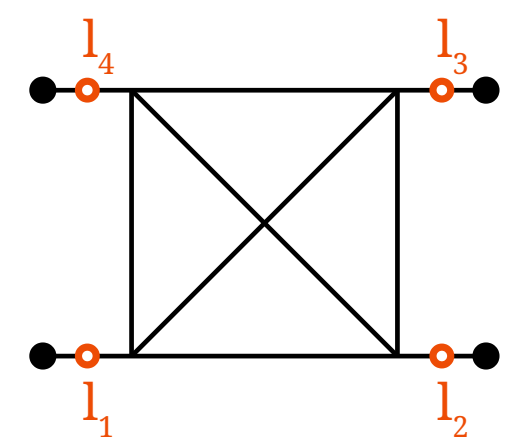

Figure A3: Inspection location setup that leads to a challenging optimization problem. The lines denote roads, the solid black circles origins and destination, and the hollow orange circles potential inspection locations.

induced by the budget constraint become less significant if a large budget is considered so that a potential remainder of the budget becomes insignificant. For example, in all scenarios with a budget above 30 units considered in this paper, we reached a solution with at least $98 \%$ optimality within minutes. Furthermore, issues induced by budget constraints can be mitigated by investigating alternative scenarios with slightly adjusted budgets.

Scenarios with unfavourable relationships between potential inspection locations can be expected in real-world applications. Note that the issue with the setup in Figure A3 persists if the roads connecting the potential inspection locations have a shape different from the road pattern drawn in the figure. Furthermore, the depicted situation may refer to a portion of the road network only, with multiple origins and destinations connected to each of the depicted origin/destination vertices. In fact, situations such as the considered one could appear multiple times in a road network. Therefore, the considered challenges do not only occur in scenarios in which inspections are restricted to locations close to origins and destinations.

Nonetheless, invasion patterns frequent in real-world scenarios reduce the prevalence of such unfavourable inspection station relationships. As short distance dispersal of invasive species is typically more likely than long-distance dispersal, invaded habitat patches form clusters so that the inflow of potentially infested vectors, such as boaters, comes from specific directions only. For example, high-risk boaters enter BC through the southern and eastern border only. Therefore, it is often possible to identify inspection location configurations in which only few high-risk boaters 
pass multiple operated inspection stations. This simplifies optimization of the inspection policy. Greater optimization challenges can be expected if origins and destinations are intermixed. 